\title{
Real-Time TD-DFT with Classical Ion Dynamics: Methodology and Applications
}

\section{Citation}

Kolesov, Grigory, Oscar Grånäs, Robert Hoyt, Dmitry Vinichenko, and Efthimios Kaxiras. 2015. "Real-Time TD-DFT with Classical Ion Dynamics: Methodology and Applications." Journal of Chemical Theory and Computation 12 (2): 466-76. https://doi.org/10.1021/acs.jctc.5b00969.

\section{Permanent link}

http://nrs.harvard.edu/urn-3:HUL.InstRepos:41384036

\section{Terms of Use}

This article was downloaded from Harvard University's DASH repository, and is made available under the terms and conditions applicable to Other Posted Material, as set forth at http:// nrs.harvard.edu/urn-3:HUL.InstRepos:dash.current.terms-of-use\#LAA

\section{Share Your Story}

The Harvard community has made this article openly available.

Please share how this access benefits you. Submit a story.

Accessibility 


\title{
Real-Time TD-DFT with Classical lon Dynamics: Methodology and Applications
}

\author{
Grigory Kolesov, ${ }^{\dagger}$ Oscar Grånäs, ${ }^{\dagger, \dagger}$ Robert Hoyt, ${ }^{\S}$ Dmitry Vinichenko, ${ }^{\|}$and Efthimios Kaxiras ${ }^{*}, \dagger, \S$ \\ †'John A. Paulson School of Engineering and Applied Sciences, Harvard University, Cambridge, Massachusetts 02138, United States \\ ${ }^{*}$ Department of Physics and Astronomy, Division of Materials Theory, Uppsala University, Box 516, SE-75120 Uppsala, Sweden \\ ${ }^{\S}$ Department of Physics, Harvard University, Cambridge, Massachusetts 02138, United States \\ "Department of Chemistry and Chemical Biology, Harvard University, Cambridge, Massachusetts 02138, United States
}

ABSTRACT: We present a method for real-time propagation of electronic wave functions, within time-dependent density functional theory (RT-TDDFT), coupled to ionic motion through mean-field classical dynamics. The goal of our method is to treat large systems and complex processes, in particular photocatalytic reactions and electron transfer events on surfaces and thin films. Due to the complexity of these processes, computational approaches are needed to provide insight into the underlying physical mechanisms and are therefore crucial for the rational design of new materials. Because of the short time step required for electron propagation (of order $\sim 10$ attoseconds), these simulations are computationally very demanding. Our methodology is based on numerical atomic-orbital-basis sets for computational efficiency. In the computational package, to which we refer as TDAP-2.0 (Time-evolving Deterministic Atom Propagator), we have implemented a number of important features and analysis tools for more accurate and efficient treatment of large, complex systems and time scales that reach into a fraction of a picosecond. We showcase the capabilities of our method using four different examples: (i) photodissociation into radicals of opposite spin, (ii) hydrogen adsorption on aluminum surfaces, (iii) optical absorption of spin-polarized organic molecule containing a metal ion, and (iv) electron transfer in a prototypical dyesensitized solar cell.

\section{INTRODUCTION}

Devices like photovoltaics, photocatalytical platforms, and batteries involve electronic excited states produced by light or other external agents and are essential in many technological applications such as renewable energy capture and storage. Excited state dynamics of electrons is also important in many biological processes such as photosynthesis and radiation damage of biomolecules like DNA. Understanding the operation of the devices and biological systems mentioned here, and contributing to their further development and enhanced operation, requires accurate modeling of physical processes that involve excited electronic states. This requires simulations that typically involve a few hundred atoms, which participate in an essential way in the process of electron excitation and in the time evolution of the excited state of the system. In addition, the time evolution of interesting processes may require picosecond time scales $\left(10^{-12} \mathrm{~s}\right)$ or longer, roughly 4-6 orders of magnitude larger than the time scale of electronic motion, which is of order a few attoseconds $\left(10^{-18} \mathrm{~s}\right)$.

In principle, simulations of these processes should employ the full many-body wave function of the electronic and ionic system for the entire time interval of interest, but this is clearly beyond any existing computational capabilities. Thus, reason- ably accurate and efficient approximate computational methods are required to make progress in this field. To this end, a number of mixed quantum-classical methods have been developed over the past few decades. One of the most popular is the fewest-switches surface hopping approach, introduced by Tully. ${ }^{1}$ In this approach the system of interest is simulated as a swarm of trajectories in which ions moving according to classical equation of motion are allowed to stochastically hop between potential energy surfaces as determined by probabilities derived from nonadiabatic coupling vectors and electronic populations of corresponding states. Another popular and computationally less expensive approach is the mean-field classical (usually referred to as "Ehrenfest") ion dynamics in which the effects of coherent electron time-propagation on the ions as expressed by the ionic forces are taken into account in an averaged way (see ref 2 for comparison of the two methods). The computational cost of these approaches can be further reduced by employing fast, parametrized electronic structure methods $^{3,4}$ or semiempirical methods. ${ }^{5-7}$ Both types of approaches are available as part of computational packages,

Received: October 10, 2015 
for example, the surface-hopping approach and its extensions in NewtonX, ${ }^{8}$ ANT, ${ }^{9}$ SHARC, ${ }^{10} \mathrm{JADE}^{11}$ and PYXAID. ${ }^{12,13}$ The first four in this list are aimed at nonperiodic systems and rely on accurate, but computationally expensive, quantum-chemistry methods to obtain potential energy surfaces and nonadiabatic couplings (although some support semi-empirical methods), while PYXAID currently employs plane-wave basis sets and propagates the electronic system along ground-state ionic trajectories, which allows it to treat large periodic systems. The Ehrenfest dynamics approach is implemented, for example, in real-space grid codes GPAW ${ }^{14}$ and OCTOPUS. ${ }^{15}$

We have already reported our initial effort toward the goal of simulating nonadiabatic dynamics of large, periodic systems for extended time scales, with a real-time version of timedependent density functional theory (RT-TDDFT) and Ehrenfest ion dynamics. ${ }^{16}$ This method was used to successfully simulate a number of photoactivated reactions including frequency-dependent splitting of simple molecules, ${ }^{16}$ electron transfer processes in dye-sensitized solar cells, ${ }^{17}$ and light absorption and energy dissipation in eumelanin molecules. ${ }^{18}$

In the present work, we report the implementation of a number of key improvements to our earlier formulation of the method $^{16}$ and several new analysis tools. These include improvements of the time propagation and parallelization algorithms, allowing for picosecond-scale simulations of large systems with hundreds of atoms. For example, we have already benchmarked our method on an 800-atom system. We also have added the ability to perform nonadiabatic DFT $+U$ simulations, which allows modeling electron dynamics in transition metal oxides or metal-organic complexes with better accuracy, while keeping computational costs tractable. Our method is built on top of the SIESTA package, ${ }^{19}$ which uses numerical atomic-orbital-basis sets to represent the electronic wave functions, which leads to extremely fast calculations while providing reasonably accurate results. Our benchmarks have demonstrated that our method is able to simulate excited-state dynamics at computational costs that are orders of magnitude lower than similar, publicly available methods that employ different basis sets. As an example of the application of the recently added features, we have simulated the photocatalytic dehydrogenation reaction of methoxy on a rutile titanium dioxide surface and for the first time gained detailed insight into the reaction dynamics that reveal a host of interesting features. $^{20}$ The new analysis tools added to TDAP-2.0 include Hirshfeld charge partitioning ${ }^{21}$ and the calculation of local dipole contributions; both tools are of great value for the qualitative analysis of charge dynamics and the response of systems to perturbing electric fields.

\section{METHODOLOGY}

2.1. Real-Time TDDFT and Classical Ion Dynamics. To simulate the nonadiabatic dynamics of coupled electron-ion systems, we use a combination of RT-TDDFT and molecular dynamics, handling the ionic degrees of freedom classically. ${ }^{14,15,22-25}$ We propagate the electronic density according to the time-dependent Kohn-Sham equations: ${ }^{26}$

$$
i \frac{\partial \phi_{n}(t)}{\partial t}=\hat{H}_{K S}[\rho](t) \phi_{n}(t), \rho(\mathbf{r}, t)=\sum_{n=1}^{N}\left|\phi_{n}(\mathbf{r}, t)\right|^{2}
$$

where $\hat{H}_{K S}$ is the Kohn-Sham Hamiltonian, $\phi_{n}$ is Kohn-Sham (KS) single particle wave function, and $\rho(\mathbf{r}, t)$ is the electronic density. Here and in the following, we use atomic units. The iterative solution of eq 1 for the time-dependent KS (TDKS) wave functions is given by

$$
\phi_{n}(t)=\hat{U}\left(t, t_{0}\right) \phi_{n}\left(t_{0}\right), \hat{U}\left(t, t_{0}\right)=\hat{T} \exp \left(-i \int_{t_{0}}^{t} \hat{H}_{K S}\left(t^{\prime}\right) d t^{\prime}\right)
$$

with the wave functions $\phi_{n}\left(t_{0}\right)$ known at some initial time $t_{0}$, and $\hat{U}$ being the time evolution operator with $\hat{T}$ the timeordering operator. In our numerical scheme, we use finite time steps $\Delta t$ and the evolution operator $\hat{U}$ is approximated with the second-order Magnus expansion: ${ }^{27}$

$$
\hat{U}(t+\Delta t, t) \approx \exp \left\{-i \Delta t \hat{H}_{K S}\left(t+\frac{\Delta t}{2}\right)\right\}
$$

We employ the localized basis set approach implemented in SIESTA, ${ }^{19}$ using the LDA $+U$ branch of SIESTA ${ }^{28}$ as a base for our code. In the finite, nonorthonormal basis set, the TDKS equations are approximated by

$$
i \frac{\partial \mathbf{c}_{n}}{\partial t}=\mathbf{S}^{-1}(\mathbf{H}-i \mathbf{P}) \mathbf{c}_{n}
$$

where $\mathbf{c}_{n}$ are the complex expansion coefficients of the $n$th wave function $\psi_{n}=\sum_{i} c_{n}^{i} \chi_{i}, \chi_{i}(\mathbf{r}, \mathbf{R}(t))$ is the basis function ( $\mathbf{R}$ is the vector of ionic coordinates). The matrix elements of the overlap matrix $\mathbf{S}$, matrix $\mathbf{P}$ due to the motion of the basis functions, and Hamiltonian matrix $\mathbf{H}$ are given by

$$
S_{i j}=\left\langle\chi_{i} \mid \chi_{j}\right\rangle, P_{i j}=\left\langle\chi_{i} \mid \frac{d}{d t} \chi_{j}\right\rangle, H_{i j}=\left\langle\chi_{i}\left|\hat{H}_{K S}\right| \chi_{j}\right\rangle
$$

The time evolution operator is calculated according to

$$
\hat{U}(t+\Delta t, t) \approx \exp \left\{-i \Delta t\left[\mathbf{S}_{1 / 2}^{-1}\left(\mathbf{H}_{1 / 2}-i \mathbf{P}_{1 / 2}\right)\right]\right\}
$$

where the subscript $1 / 2$ indicates matrices taken at the half time step:

$$
\mathbf{H}_{1 / 2}=\frac{1}{2}[\mathbf{H}(t)+\mathbf{H}(t+\Delta t)] \approx \mathbf{H}\left(t+\frac{\Delta t}{2}\right)
$$

Each time propagation step is iterated to self-consistency as described in ref 27 . We use the Lanczos iterative method ${ }^{27,29}$ and the Padé rational polynomial approximation of arbitrary degree (typically from $3 / 3$ to $6 / 6$ ) to obtain the general matrix exponential in eq 3 . The latter was augmented with a standard "scaling and squaring" method to improve stability and to achieve high numerical accuracy. ${ }^{30}$ On large systems, our testing demonstrated superior performance and scaling of the Pade method relative to the Lanczos iterative method.

The classical ions are propagated according to Newton's equations: $M_{J} \ddot{\mathbf{R}}_{J}=\mathbf{F}_{J}$, where $J$ is ion index. In our current implementation, we follow the approach described in refs 14, 22 , and 23 to obtain the forces $\mathbf{F}_{J}$. This is done by setting $d E_{t o t} /$ $d t=0$, that is, by requiring conservation of the total energy $E_{t o t}$. This results in energy-conserving (EC) forces on the ions, which are given by

$$
\begin{aligned}
\mathbf{F}_{J} & =-\nabla_{\mathbf{R}_{J}} \sum_{K \neq J} \frac{Z_{J} Z_{K}}{\left|\mathbf{R}_{J}-\mathbf{R}_{K}\right|}-\sum_{n} f_{n}\left[\mathbf{c}_{n}^{*}\left(\nabla_{\mathbf{R}_{j}} \mathbf{H}\right) \mathbf{c}_{n}\right. \\
& -\sum_{i, j} c_{n}^{* i}\left\langle\chi_{i}\left|\nabla_{\mathbf{R}_{J}}\left(V_{H}+V_{x c}\right)\right| \chi_{j}\right\rangle c_{n}^{j} \\
& \left.-\mathbf{c}_{n}^{*}\left(\mathrm{HS}^{-1} \mathbf{d}^{J}+\text { h.c. }\right) \mathbf{c}_{n}\right]
\end{aligned}
$$


where $V_{H}$ and $V_{x c}$ are the Hartree and exchange-correlation potentials, respectively, and $\mathbf{d}^{J}$ is the vector matrix $\mathbf{d}_{i j}^{J}=$ $\left\langle\chi_{i}\left|\nabla_{\mathbf{R}_{l}}\right| \chi_{j}\right\rangle$. In the case of adiabatic dynamics, the last term in brackets in eq 4 reduces to the ordinary Pulay force term, ${ }^{19,31}$ $f_{n} \varepsilon_{n} \mathbf{c}_{n}\left(\nabla_{\mathbf{R}_{f}} \mathbf{S}\right) \mathbf{c}_{n}$, with $\varepsilon_{n}$ being the eigenvalue of the $n$th KS wave function.

The forces on classical ions in a mixed quantum-classical system were derived in the most rigorous way by Kunert and Schmidt. ${ }^{24}$ This was done by considering a quantum action for the electrons, a classical action for the ions, and requiring the total action to be stationary. It resulted in additional velocitydependent and double-derivative higher order correction terms to the EC forces in eq 4 (see eqs 34 and 35 in ref 24). Following refs 14 and 23, however, we neglect these terms in our current implementation of the dynamics. Both of these methods, EC and the stationary quantum-classical action method, differ from the traditional Ehrenfest method (see refs 32-34 and references therein) only in the introduction of correction or "constraint" forces resulting from the incomplete basis set. ${ }^{22,24}$ Despite the simplicity of the Ehrenfest method, it is expected to work well for systems with high densities of excited states such as surfaces and bulk systems where a single reaction path dominates. The latter is often the case in practical applications, for instance, in highly selective photocatalytic systems.

2.1.1. Energy Conservation in Excited Ozone. To demonstrate the importance of using nonadiabatic EC forces as expressed in eq 4 instead of adiabatic forces, we discuss a simple example, the excited ozone molecule. We thermalized the ozone molecule at $200 \mathrm{~K}$ with standard molecular dynamics, excited it into the first electronic excited state, and then simulated the dynamics of the system using the time step $\Delta t=24.2$ as. To see how significant the corrections in the equation of motion (eq 4) are, we also simulated the dynamics with a simpler method, where forces on ions were calculated assuming quasi-adiabatic (QA) behavior of the wave functions, that is, assuming that the $\mathrm{KS}$ wave functions deviate insignificantly from the eigenstates of the KS Hamiltonian. The trajectory and energy conservation in both simulations are presented in Figure 1. The top panel shows the difference in EC and QA excited-state trajectories, which are close in the two approaches for four vibrational periods but diverge after this time interval. At 1 ps the trajectories are clearly different, both in amplitude and frequency of the vibrations. The bottom panel of Figure 1 demonstrates that the total energy in the simulation with QA forces starts to deviate significantly after about two vibrational periods and eventually diverges (error in energy $>3$ $\mathrm{eV}$ ). In the simulation with EC forces the total energy essentially stays constant (maximum deviation of $\sim 0.2 \mathrm{meV}$ ) even after one picosecond.

2.2. The LDA $+U$ Method in RT-TDDFT. RT-TDDFT is in principle capable of providing accurate excited states for arbitrary compounds. ${ }^{35}$ This, however, depends on how well the electronic density is described by the exchange-correlation functional used. Note that it does not necessarily rely on the unoccupied KS states of the ground state density, as is common in GW implementations and linear response TDDFT. This means that it suffers less from deficiencies introduced by considering individual KS quasi-particle states, as absorption and reflectance spectra are calculated from the time-dependent density response. In practice, several caveats remain, particularly errors introduced by self-interaction in compounds with
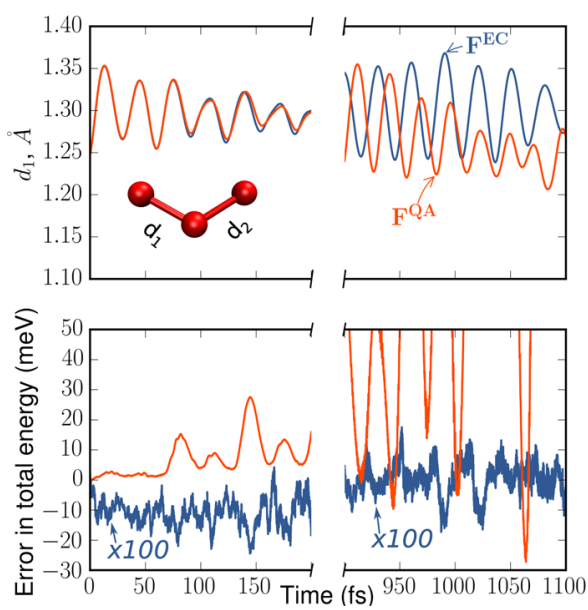

Figure 1. Excited-state dynamics of ozone. Top: Time dependence of bond length $d_{1}$ (see inset) in simulation with quasi-adiabatic (QA) forces (red line) and with energy-conserving (EC) forces (blue line). Bottom: Energy conservation as a function of time in the two simulations. The blue line has been scaled up by a factor of 100 to make it visible on the same scale as the red line.

localized electrons in partially filled bands, such as compounds that contain transition metals and lanthanides. For ground-state DFT, a partial remedy to this was introduced with the LDA+U method. ${ }^{36,37}$ The method was originally formulated by introducing the $U$ term, the effective orbital-dependent Coulomb interaction, from the Hubbard model. When solved as a static mean-field, such as screened Hartree-Fock, this is readily applied to the KS Hamiltonian. The $\mathrm{LDA}+U$ method has been refined over the years to account for the double counting in different limits ${ }^{36,38}$ and interpolation in between these limits, ${ }^{39}$ to include full noncollinearity, ${ }^{40}$ and has recently been reformulated as a parameter-free Hubbard density functional. ${ }^{41-44}$ In the linear response version of TDDFT, it has been used to study the Frenkel exciton in $\mathrm{NiO} .{ }^{45}$

RT-TDDFT is often employed using a ground state DFT exchange-correlation functional, assuming that the exchange and correlation is adiabatically changed from the previous timestep. The $L D A+U$ method fits very well into this scheme since the Hartree-Fock interaction and the adiabatic exchangecorrelation kernel are instantaneous. Since the $U$ term is applied to remedy deficiencies in the exchange correlation functional for localized electrons, it is only applied for a subset of orbitals such as open $d$ - or $f$-shells. This makes the method substantially faster than full-range hybrid functionals, where the long-ranged Hartree-Fock (HF) contribution requires a considerable amount of computational effort. The localized description is achieved by projecting the density matrix onto the local basis functions $\chi_{i}$ spanning the particular subset of interest. Within the local subspace a fully rotationally invariant ${ }^{46,47} \mathrm{HF}$ correction can be written as

$$
E_{\mathrm{HF}}=\frac{1}{2} \sum_{i j l k}\left[\rho_{i k} \rho_{j l}-\rho_{i l} \rho_{j k}\right] U_{i j k l}
$$

where $\rho_{i j}=n_{i} n_{j}\left\langle\chi_{i} \mid \chi_{j}\right\rangle$ is an element of the local density matrix, and $U_{i j k l}=\left\langle\chi_{i} \chi_{j}|g| \chi_{k} \chi_{l}\right\rangle$ with $g$ being the screened Coulomb interaction.

Since part of the screened HF potential is already included in the DFT effective potential, a correction is needed to avoid double counting the interactions. To this end, we have 
implemented three different approaches. The first prescription is derived by considering a localized state with integer occupation. Assuming that, without orbital polarization, the energy expression should reduce to that of LDA, one can derive the energy and potential corrections that have to be removed. ${ }^{48}$ This approach is referred to as the fully localized limit (FLL). We define the charge, $n$, and magnetic moments, $\mathbf{m}$, from the density matrix through

$$
\begin{aligned}
& n=\operatorname{Tr} \rho \\
& \mathbf{m}=\operatorname{Tr} \boldsymbol{\sigma} \rho
\end{aligned}
$$

where the Pauli spin matrices, $\boldsymbol{\sigma}$, act on the spin degree of freedom only, $\sigma_{i j}=\delta_{m_{i} m_{j}} \boldsymbol{\sigma}_{s_{i} j_{j}}$ Using these quantities, the double counting term to subtract is

$$
E_{\mathrm{dc}}^{\mathrm{FLL}}=\frac{1}{2}\left[U n(n-1)-J n\left(\frac{n}{2}-1\right)-\frac{J}{2} \mathbf{m} \cdot \mathbf{m}\right]
$$

The second approach, derived for metallic systems, is commonly referred to as the around mean-field (AMF) approach. The rationale is that common exchange-correlation functionals typically give a good description of the spherically averaged charge and magnetization densities. The AMF approach is defined by subtracting the averaged charge and magnetization contributions from the density matrices. The resulting new auxiliary density matrix

$$
\tilde{\rho}_{i j}=\rho_{i j}-\left(\delta_{i j} n+\boldsymbol{\sigma}_{i j} \cdot \mathbf{m}\right) / D
$$

where $D$ is the number of spin-orbitals and is then used in the energy expression eq 5, giving the AMF-double-counting corrected $\mathrm{LDA}+U$ energy term

$$
E_{\mathrm{dc}}^{\mathrm{AMF}}=\frac{1}{2} \sum_{i j l k}\left[\tilde{\rho}_{i k} \tilde{\rho}_{j l}-\tilde{\rho}_{i l} \tilde{\rho}_{j k}\right] U_{i j k l}
$$

The respective potential contributions are defined as

$$
V_{i j}=\frac{\delta E_{U}}{\delta \rho_{j i}}=\sum_{k l}\left[U_{j k i l}-U_{j k l i}\right] \rho_{k l}
$$

which for the FLL and AMF corrections lead to

$$
\begin{aligned}
V_{i j}^{\mathrm{FL}} & =V_{i j}-\left[\frac{U(2 n-1)}{2}-\frac{J(n-1)}{2}\right] \delta_{i j}+\frac{J \mathbf{m} \cdot \boldsymbol{\sigma}_{i j}}{2} \\
V_{i j}^{\mathrm{AFM}} & =\sum_{k l}\left[U_{j k i l}-U_{j k l i}\right] \tilde{\rho}_{k l}
\end{aligned}
$$

Although most calculations use one of these limits, the real system lies somewhere in between. This led Petukhov et al. ${ }^{39}$ to develop the third approach, which is an interpolation scheme (INT) between these two limits. AMF always gives a negative contribution to the energy, whereas FLL always gives a positive contribution. DFT is regarded to have a good total energy, but incorrect potential. On the basis of this argument, one defines an interpolation parameter $\alpha$, determined in a self-consistent way, to minimize the energy contribution from the double counting correction:

$$
\alpha=\frac{D \operatorname{Tr} \tilde{\rho}^{2}}{D n-n^{2}-m^{2}}
$$

This correction results in the potential

$$
V^{\mathrm{INT}}=\alpha V_{i j}^{\mathrm{FLL}}+(1-\alpha) V_{i j}^{\mathrm{AFM}}
$$

It is possible to make significant simplifications by assuming a spherically symmetric Coulomb term, which together with the FLL double counting, gives ${ }^{49}$

$$
V_{i j}^{\text {sym }}=(U-J)\left[\frac{\delta_{i j}}{2}-\rho_{j i}\right]
$$

The SIESTA LDA $+U$ branch implements this latter approach, ${ }^{28}$ and our implementation of $L D A+U$ is derived from it.

2.3. Dielectric Properties from RT-TDDFT. In nonperiodic systems, the dielectric function is readily obtained from analyzing the response of the system to an external electric field. ${ }^{5-52}$ The system is perturbed by a short, low intensity external electric field pulse, with duration shorter than any of the expected density response times. Subsequently the time-dependent dipole polarization of the system is analyzed to obtain the dielectric properties. The time dependent oscillation of the dipole moment is calculated through

$$
p_{j, k}(t)=\operatorname{Tr}\left[\mathbf{D}^{k} \rho(t)\right]
$$

where $j$ the indicates direction of an applied electric field (see below) $\hat{\mathbf{e}}_{j}=\hat{\mathbf{x}}, \hat{\mathbf{y}}, \hat{\mathbf{z}}, k$ indicates the direction of measurement, and $\mathbf{D}$ is the transition dipole tensor operator defined through

$$
\mathbf{D}_{\mu \nu}^{k}=\left\langle\chi_{\mu}(\mathbf{r})\left|\hat{e}_{k} \cdot \mathbf{r}\right| \chi_{\nu}(\mathbf{r})\right\rangle
$$

This in turn is related to the polarizability tensor $\alpha(t)$ through

$$
p_{j, k}(t)=\int_{-\infty}^{t} \alpha_{j, k}\left(t-t^{\prime}\right) E_{j}\left(t^{\prime}\right) d t^{\prime}
$$

where $E_{j}(t)$ is the $j$ th component of an external electric field. By assuming that $\mathbf{E}(t)$ has the form $\mathbf{E}(\mathrm{t})=E_{0} \delta\left(t-\mathrm{t}_{0}\right) \hat{\mathbf{e}}_{j}$, after Fourier transforming, we obtain

$$
\alpha_{j, k}(\omega)=\frac{p_{j, k}(\omega)}{E_{0}}
$$

The absorption cross-section $\sigma(\omega)$ is given in terms of $\alpha(\omega)$ as

$$
\sigma_{j, k}(\omega)=\frac{4 \pi \omega}{c} \operatorname{Im}\left[\alpha_{j, k}(\omega)\right]
$$

From this, the dipole strength function (absorption spectrum) is calculated as

$$
S(\omega)=\frac{1}{3} \operatorname{Tr}[\sigma(\omega)]
$$

2.4. Excitation Classification Using Density Partitioning. One drawback of RT-TDDFT compared to orbitaldependent methods is that the orbitals of the initial and final states are not comparable due to the nonadiabatic propagation. Analysis of the excitations are therefore complicated by the lack of initial and final state information. For instance, it is difficult to address questions like "To what extent is the excitation localized and where?" or "What is the amount of charge transfer?" This information is crucial for the design of materials relying on resonant excitations or fast charge transfer. To address this, we have implemented the density partitioning scheme following Hirshfeld, ${ }^{21}$ through which we can calculate the time-dependent dipolar contribution from displacing charge between atoms (charge transfer) and displacing charge within the atoms (local excitations). The Hirshfeld scheme is 
particularly attractive as it avoids artificial sharp boundaries between atoms, relying on a weight function which defines to what extent a region belongs to a certain atom. The weight function $w_{i}$ for atom $i$ at a point $(\mathbf{r}, t)$ is determined by calculating the fraction of the atomic density $\rho_{i}^{\text {Atom }}$ that contributes to a reference system of overlapping atomic densities (the so-called "Harris" density, $\rho^{\text {Harris }}$ ),

$$
w_{i}(\mathbf{r}, t)=\rho_{i}^{\text {Atom }}(\mathbf{r}, t) / \rho^{\text {Harris }}(\mathbf{r}, t)
$$

The self-consistent field optimized charge density, $\rho^{\mathrm{SCF}}$, is then partitioned into a density of bonded atoms, $\rho^{\mathrm{BA}}$, by the corresponding weights:

$$
\rho_{i}^{\mathrm{BA}}(\mathbf{r}, t)=w_{i}(\mathbf{r}, t) \rho^{\mathrm{SCF}}(\mathbf{r}, t)
$$

This has proven to be one of the most reliable, basis-set independent, and most chemically sensible ${ }^{53}$ ways to partition a molecule. From this, we define the atomic deformation density from

$$
\delta \rho_{i}(\mathbf{r}, t)=\rho_{i}^{\mathrm{BA}}(\mathbf{r}, t)-\rho_{i}^{\mathrm{Atom}}(\mathbf{r}, t)
$$

The net atomic charge is evaluated by integrating over the fragment, subtracting the nuclear charge

$$
q_{i}(t)=-\int_{V} \rho_{i}^{\mathrm{BA}}(\mathbf{r}, t) \mathrm{d} \mathbf{v}+Z_{i}
$$

where the integral is over the entire volume of the cell; in practice, the integrand is zero outside the range of the numeric atom-centered basis functions. This allows us to calculate the charge transfer contribution (CT) to the polarization oscillations, $\mathbf{p}_{i}^{\mathrm{CT}}$, from the charge redistribution and the distance $\mathbf{r}^{\prime}$ to the system's center of mass.

$$
\mathbf{p}_{i}^{\mathrm{CT}}(t)=q_{i}(t) \mathbf{r}^{\prime}
$$

The local contribution, $\mathbf{p}^{\text {LOC }}$, for each fragment is evaluated by integrating the dipole operator over the deformation density

$$
\mathbf{p}_{i}^{\mathrm{LOC}}(t)=-\int_{V} \mathbf{r} \delta \rho_{i}(\mathbf{r}, t) \mathrm{dv}
$$

The contribution to the absorption spectra from charge transfer excitations $\left[S^{\mathrm{CT}}(\omega)\right]$ and local excitations $\left[S^{\mathrm{LOC}}(\omega)\right]$ are now calculated in accordance with the spectra from the total dipole contribution (eq 22).

2.5. Excited States from $\Delta$-SCF. In order to model electronic excited states we have implemented the $\Delta$-SCF approach, ${ }^{54-57}$ with the excitation modeled by a singly excited KS Slater determinant or a linear combination of such determinants. The standard approach within DFT for the excited states is linear-response TDDFT. ${ }^{58}$ This has been shown to be a reliable approach for small systems but often fails to describe charge transfer states in larger systems with commonly used exchange-correlation functionals. ${ }^{59,60}$ The many-body perturbation theory approaches, such as the GW and Bethe-Salpeter equation methods ${ }^{61}$ or quantum chemistry methods, cannot be combined with the RT-TDDFT propagation scheme and are often impractical for computation of excited states in large systems. The $\Delta$-SCF method, on the other hand, has certain advantages:

1. It is accurate in modeling the lowest-energy excitation. ${ }^{62}$

2. It can, in principle (with an exact functional), produce densities that are the densities of the true excited states. $^{63,64}$
3. Most importantly, in contrast to linear response TDDFT, the $\Delta$-SCF approach does not build upon the ground state wave functions of the KS Hamiltonian operator, but the orbitals involved are variationally optimized with the excitation.

This last property of the $\Delta$-SCF method allows a correct description of charge-transfer states. ${ }^{64,65}$ Recent benchmarking has shown that the accuracy of the $\Delta$-SCF method can even be on par with the more sophisticated methods such as selfconsistent GW. ${ }^{66}$

We have implented three flavors of $\Delta$-SCF. The first is a basic $\Delta$-SCF scheme, where occupation numbers of KS orbitals are altered from the zero-temperature Fermi distribution to promote an electron from a single occupied orbital to a single unoccupied one. This is done at each step of the SCF loop; the indices of the relevant pair of orbitals are kept fixed.

The second method is similar, but the indices are not fixed. At each SCF step the indices are selected by finding the orbitals that most closely resemble the spatial character of a pair of initially chosen ground state orbitals. This is similar to the method proposed in ref 65 . Our tests show that this simple modification of the basic $\Delta$-SCF scheme speeds up convergence by as much as a factor of 4 in the case of high density of states, which is the usual case in surface and bulk systems.

The third is the linear expansion $\Delta$-SCF method developed by Gavnholt et al. ${ }^{57}$ Modeling the excited states of adsorbed molecules is often of interest, for example, in studying photocatalytic properties of metals. However, metallic systems have a range of states with similar character, none of which may closely resemble a particular adsorbate state of interest. Following Gavnholt et al., we use a generalization of our second $\Delta$-SCF method to allow the highest-energy state to be a linear combination of the unoccupied KS states in conjuction with Fermi smearing, which produces the following electronic density

$$
\begin{aligned}
\rho(\mathbf{r}, t) & =\sum_{n=1}^{M} f_{N-1}\left(\epsilon_{n}\right)\left|\psi_{n}(\mathbf{r}, t)\right|^{2} \\
& +\left|\sum_{n=N}^{M} \theta\left(\delta-f_{N-1}\left(\epsilon_{n}\right)\right) a_{n} \psi_{n}(\mathbf{r}, t)\right|^{2}
\end{aligned}
$$

where $f_{N-1}$ is the Fermi distribution function for $N-1$ electrons, $M$ is the total number of KS states, and $a_{n}$ are the coefficients for the highest-occupied state chosen to resemble an a priori adsorbate state as closely as possible. The $\delta$ parameter in eq 29 is chosen to be a small value, $10^{-4}$ in our calculations, to keep the linear combination state orthogonal to the other $N-1$ occupied KS orbitals without excluding negligibly occupied states from the expansion. In typical DFT calculations involving metal surfaces, this excludes only a few states at electronic temperatures of $300 \mathrm{~K}$. The adsorbate state of interest is calculated self-consistently with basic $\Delta$-SCF in a gas-phase calculation in order to provide a better account of charge reorganization. ${ }^{67}$

\section{APPLICATIONS}

We have selected a number of different physical systems to showcase new computational capabilities described above and their ability to provide insight. These include the following: (i) A photodissociation reaction in which a molecule, upon 


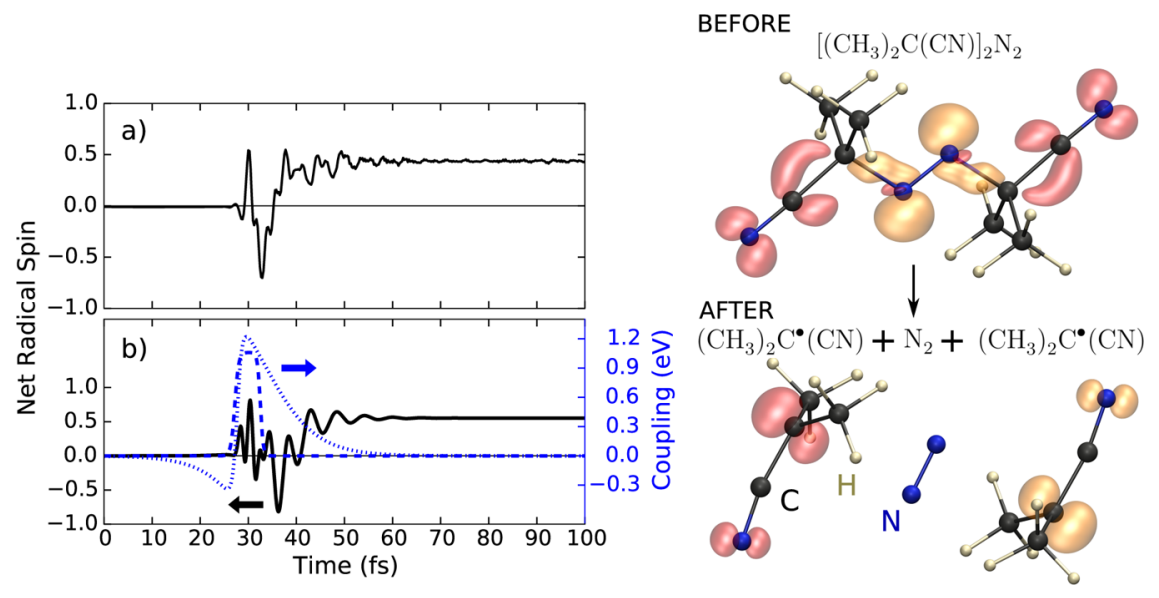

Figure 2. Net radical spin on one of the isobutyronitrile radicals calculated from (a) the nonadiabatic propagation and (b) the model Hamiltonian. In (b), the short-dash line shows the term $a(t)$ in the effective Hamiltonian, and the long-dash line shows $b(t)$. The geometry of the AIBN molecule before and after dissociation is shown on the right. The red and orange lobes are magnetization density isosurfaces of opposite sign.

excitation by light, breaks into radicals of opposite spin; this demonstrates the ability to capture reactions that happen spontaneously after excitation, and involve spin polarized reactants and products. (ii) Hydrogen absorption on aluminum surfaces; this demonstrates the ability to study reactions on metal surfaces, which involve a high density of metallic surface states, and result in interesting dynamics. (iii) Optical absorption of a metal-ion-containing, spin-polarized organic molecule; this demonstrates the ability to describe systems that involve transition-metal ions (where the $L D A+U$ approach is essential) and their interaction with light. (iv) Electron transfer in a prototypical dye-sensitized solar cell; this demonstrates the ability to describe complicated organic-inorganic combinations of systems and the coupled ion and electron dynamics responsible for their response to light excitation. This last case shows that our methodology can handle rather large systems (containing several hundreds of atoms) and simulate their behavior for hundreds of femtoseconds.

3.1. Photodissociation of Azobisisobutyronitile. Azobisisobutyronitrile (AIBN) is a common radical initiator used in polymerization. Under exposure to UV light, AIBN dissociates to form a nitrogen molecule and a symmetric pair of isobutyronitrile radicals. Linear response calculations show that the HOMO to LUMO+1 excitation closely resembles this UV transition. To simulate the photodissociation, we first determined the ground-state molecular geometry. For these calculations, we used a double- $\zeta$ polarized (DZP) basis set, collinear spin polarization, and LDA pseudopotentials. The initial condition for the dynamics was chosen to be the HOMO to LUMO+1 transition in the first spin channel using the first approach for $\Delta-S C F$ excitation, described in the previous section. The time evolution of the system was calculated using a time step of 25 as.

The dissociation proceeds as shown in Figure 2, producing a pair of isobutyronitrile radicals and a nitrogen molecule. During the dissociation, the net spin of each radical oscillates substantially before arriving at an equilibrium value. These oscillations are the result of the initial excited state, with no net magnetization on each isobutyronitrile group, gradually coupling to magnetized radical states. These magnetized states interact nonadiabatically via the DFT Hamiltonian, producing oscillations in the magnetization density.
In order to qualitatively understand this behavior, we construct a simple three-state model effective Hamiltonian $H_{\text {eff }}(t)$ from the two magnetized radical states $|\uparrow \downarrow\rangle$ and $|\downarrow \uparrow\rangle$ and the unmagnetized initial state $|\triangle S C F\rangle$. For simplicity, we model the matrix elements with sigmoidal time-dependence:

$$
\left(\begin{array}{ccc}
E & a(t) & -a\left(t-t_{0}\right) \\
a(t) & 0 & b(t) \\
-a\left(t-t_{0}\right) & b(t) & 0
\end{array}\right)
$$

where the matrix elements represent:

$$
\begin{array}{rlc}
\left\langle\Delta \mathrm{SCF}\left|H_{\text {eff }}(t)\right| \Delta \mathrm{SCF}\right\rangle & = & E=0.71 \mathrm{eV} \\
\left\langle\Delta \mathrm{SCF}\left|H_{\text {eff }}(t)\right| \uparrow \downarrow\right\rangle & = & -\left\langle\Delta \mathrm{SCF}\left|H_{\text {eff }}(t)\right| \downarrow \uparrow\right\rangle=a(t) \\
\left\langle\uparrow \downarrow\left|H_{\text {eff }}(t)\right| \downarrow \uparrow\right\rangle & = & b(t)
\end{array}
$$

The coupling to the $|\downarrow \uparrow\rangle$ state is shifted by $t_{0}=1$ fs to model the slight initial asymmetries that lead to the $|\uparrow \downarrow\rangle$ state first dominating the overall magnetization. The numerical solution for the net radical spin is calculated as the probability of being in the $|\uparrow \downarrow\rangle$ state minus that of being in $|\downarrow \uparrow\rangle$ state. A comparison between the DFT and model net radical spin, as well as the time-dependence of the $a(t)$ and $b(t)$ matrix elements, is shown in Figure 2. The initial onset of large oscillations, their amplitude, and the trailing fluctuations around $50 \mathrm{fs}$ are qualitatively well-reproduced despite the dramatic simplifications implied by the model.

3.2. Spin Transition in $\mathrm{H} / \mathrm{Al}(111)$. Adsorption of atomic $\mathrm{H}$ on metallic surfaces exhibits a phase transition in the $\mathrm{H}$ atom's net spin polarization, with square-root dependence of the net polarization on distance from the surface. ${ }^{68-72}$ This transition is a fundamentally nonadiabatic effect, dissipating roughly $200 \mathrm{meV}$ of the adsorption energy into the metal electronic degrees of freedom over the first period of $\mathrm{H}$ atom motion for the FCC (111) top site. ${ }^{69,71}$ This process offers a useful test of our method's accuracy and performance since it has been studied in detail by similar approaches, for example by Lindenblatt and Pehlke. ${ }^{71}$

We model the system representing $H$ adsorption by a $2 \sqrt{ } 3 \times$ $2 \sqrt{3}$ supercell of the $\mathrm{Al}(111)$ bulk-terminated plane. We use a single- $\zeta$ polarized (SZP) basis set, $35 \mathrm{Ry}$ real-space cutoff for the calculation of Fourier transforms, a slab consisting of six 
layers of $\mathrm{Al}$ atoms (of which the bottom two are fixed to their ideal positions while the rest of the atoms are allowed to move), collinear spin polarization, and a single k-point to sample the surface Brillouin zone. We chose these compuational parameters on purpose to check if the minimal amount of computational effort that they represent can capture the essential physics of the process. These settings closely resemble those of Lindenblatt and Pehlke. ${ }^{71}$

At several intermediate points in the simulation, the dissipated energy is calculated as the difference between the electronic energy in the nonadiabatic calculation, and the corresponding ground-state electronic energy with identical atomic positions. The $\mathrm{H}$ atom starts $4 \AA$ above the $\mathrm{Al}(111)$ top site and directly approaches the surface with an initial kinetic energy of $60 \mathrm{meV}$. The motion trail of the $\mathrm{H}$ atom colliding with the top site is shown in the inset of Figure 3, and our

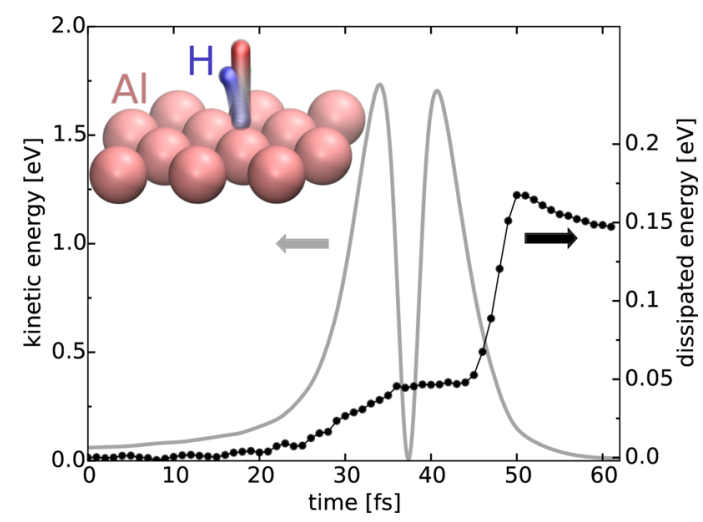

Figure 3. $\mathrm{H}$ atom kinetic energy and the total energy dissipated into the electronic degrees of freedom for $\mathrm{H}$ adsorption over the top site of the $\mathrm{Al}(111)$ surface. Inset shows the motion trail over the first $60 \mathrm{fs}$ of the $\mathrm{H}$ atom adsorbing at the $\mathrm{Al}(111)$ top site. The $\mathrm{H}$ atom's initial position is shown in red, and its position at $60 \mathrm{fs}$ is shown in blue. Only the top layer of $\mathrm{Al}$ atoms is shown for clarity.

results for the $\mathrm{H}$ atom's kinetic energy and the nonadiabatic energy dissipation in the first period of the $\mathrm{H}$ atom's motion are presented in Figure 3. The two step-like jumps in the electronically dissipated energy correspond to the $\mathrm{H}$ atom crossing the spin transition point, once on approach and again right after it collides with the $\mathrm{Al}(111)$ top site. We calculate a maximum energy of $\sim 0.17 \mathrm{eV}$ dissipated to the electronic degrees of freedom, close to the $\sim 0.18 \mathrm{eV}$ found by Lindenblatt and Pehlke. ${ }^{71}$ This dissipated energy is twice the amount transferred to the $\mathrm{Al}$ atoms' total kinetic energy, highlighting the importance that nonadiabatic effects can have on adsorption at metallic surfaces. We note that despite severe approximations such as the SZP basis set and the absence of correction for basis-set superposition error, all essential results of ref 71 are reproduced well in our simulation.

3.3. Optical Properties of Fe Porphyrin. A well-studied example of optically active structures in the visible range is the family of molecules called porphyrins. Porphyrin molecules have a long history of being used as light harvesting dyes in Grätzel solar cells. ${ }^{73,74}$ They also occur in the natural photosynthetic process. Fe porphyrin, in particular, is interesting as it is associated with the electron transfer in the respiratory chain of many animals. The optical properties of $\mathrm{Fe}$ porphyrin can therefore be used in forensics to establish the time since depositions of blood stains because they exhibit a shift in the onset of absorption upon oxidation. ${ }^{75}$ Moreover, due to its open $d$-shell, leading to a magnetic moment, the Fe porphyrin has been suggested for use in data storage applications. $^{76,77}$ We describe here the local character of the $d$-shell using the LDA+ $U$ method outlined in section 2.2. This method has been applied to calculate ground state properties by Panchmatia et al., ${ }^{77}$ showing good agreement with experiment. ${ }^{76}$ We study the absorption spectra by investigating the response of the electronic density to an electric field pulse. Similar methodology has been used to study $\mathrm{Zn}$ porphyrin, which from a computational perspective is considerably easier to handle due to the full $d$-shell of the $\mathrm{Zn}$-atom. ${ }^{51}$

When studying absorption as an impulse response, care has to be taken so that the duration of the pulse is proportionally shorter for faster expected response times and the field strength should be sufficiently small so as not to perturb the system beyond the linear regime. We model the pulse by a Gaussian field with a standard deviation of 2.4 as and a height of $0.02 \mathrm{Ry} /$ $(\hbar / e)$. The calculations are performed with a DZP basis set using the PBE exchange-correlation functional. The Hubbard $U$ and $J$ parameters are 4 and $1 \mathrm{eV}$, respectively, the same as in ref 77. We use the partitioning scheme outlined in section 2.4 to analyze the character of the excitations. In general, the polarizability of a material can only be seen as a sum of fragments in the limit of an ionic compound through the socalled Claussius Mosotti relations. In the method outlined here, we have the option to normalize the sum of the fragments to the total spectra.

In Figure 4, we see the dominant Soret peak, known to be a $\pi-\pi^{*}$ excitation, as predominantly oscillations on the outer

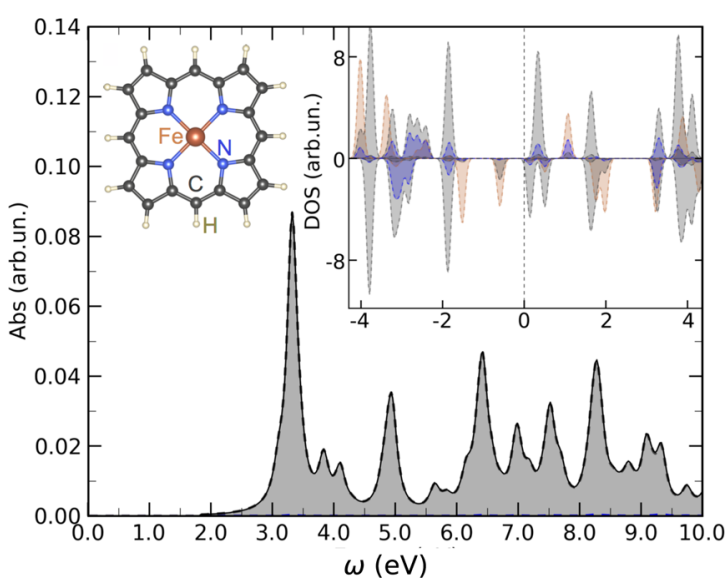

Figure 4. Optical absorption of Fe porphyrin. The Soret peak is clearly visible at $\sim 3.2 \mathrm{eV}$. The inset shows the density of states of the ground states, with different contributions colored by the atom type, as shown in the structure on the left. The total absorption spectra is a solid black line.

carbon and hydrogen atoms. The onset of absorption is in excellent agreement with experiment. The Soret peak at $3.2 \mathrm{eV}$ is slightly lower in energy than that of $\mathrm{Zn}$ porphyrin due to effects of the open $d$-shell upon the $\pi$-bonds of the outer carbon ring. By examining the ground state density of states, we find that no Fe $d$-states are expected to be significantly optically active in this energy range, as they are either Laporte and/or spin forbidden in the region close to the gap. Instead, as confirmed by the Hirshfeld partitioning, the excitations are exclusively on the carbon and hydrogen atoms. 
3.4. Perylene on the Rutile $\mathrm{TiO}_{2}$ Surface. The method we presented here is intended to be used on large systems such as surfaces and interfaces which are composed of several hundreds of atoms. In this final example, we provide a brief demonstration of our method with such a system; we study electron transfer from a chromophore molecule to a rutile $\mathrm{TiO}_{2}$ (110) surface.

The ionic motion can have a substantial impact on effects of electron transfer. It is well known that in rutile titania along the (110) direction electron carriers have an effective mass of 10$15 m_{e}$ due to the formation of small polarons. ${ }^{78-80}$ Here, we demonstrate the effect of electron self-trapping on electron transfer in a dye-sensitized solar cell system. As a chromophore, we chose perylene, a relatively simple and thoroughly studied molecule in ultrafast pump-probe experiments. ${ }^{81,82}$ For simplicity, we chose carboxylic acid as the anchor group of the molecule to the surface. We model the $\mathrm{TiO}_{2}$-rutile (110) surface by a slab consisting of four atomic layers along the crystallographic direction, as shown in Figure 5. The number of

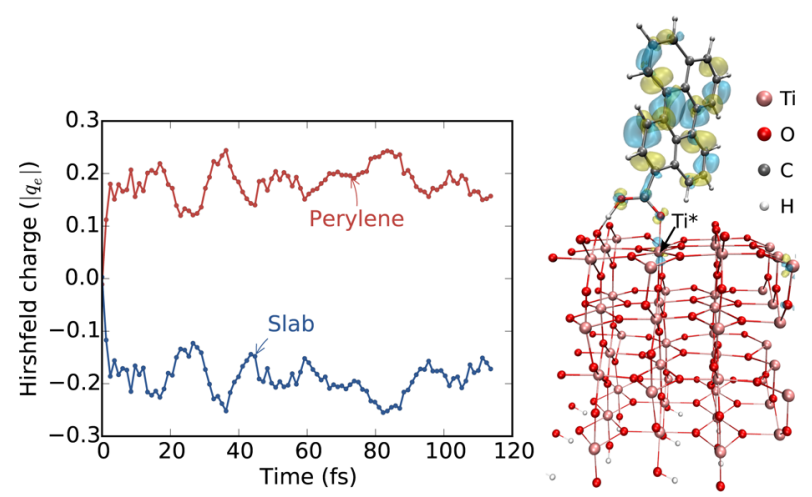

Figure 5. Left: Evolution of the charge, as calculated by the Hirshfeld partitioning method, on the perylene molecule and on the slab representing the $\mathrm{TiO}_{2}$ surface after excitation. Right: Simulation cell at the time of the excitation. The electronic excited state is plotted as the difference between the excited and ground state charge densities computed with the same ionic coordinates; blue isosurface correspond to the negative charge (electron) and yellow ones to the positive charge (hole).

layers was chosen based on earlier convergence studies. ${ }^{83}$ The dangling bonds at the bottom of the slab are passivated with hydrogen atoms. The total number of atoms in the simulation cell is 197; during the dynamics, the bottom of the slab (48 atoms) is kept fixed with the interatomic distances equal to the corresponding bulk values. In our simulation, the concentration of the adsorbed perylene molecules on the titania surface roughly corresponds to the one used in experiments $(0.18 \mathrm{ML}$, see ref 81 ). In previous studies, a $D F T+U$ or higher level of treatment was necessary to properly describe electron-ion dynamics in titania, in particular to capture the formation of small polarons. ${ }^{84,85}$ Here, we use $\mathrm{PBE}+U$ with $U=4.2 \mathrm{eV}$. This is a typical value for titania and has been confirmed previously in convergence studies. ${ }^{20,83}$ We use a DZP basis set and a collinear spin-polarized setup, and we sample the Brillouin zone by the $\Gamma$ point.

In dye-sensitized solar cell designs such as the one described here, the occupied states of the chromophore do not interact significantly with the states of the semiconductor. As a result, the probability of direct electron injection from the chromophore into the semiconductor is negligibly small, and once the electron has been transferred to the semiconductor, its direct recombination with the hole in the chromophore is blocked. Therefore, to study the dynamics of the electron transfer from perylene to the titania surface, we ignored lower energy perylene-to-surface charge transfer states and chose the excited state with both the electron and hole localized on perylene (Figure 5). This state has an excitation energy of 2.4 $\mathrm{eV}$ and was found by using the second type of the $\Delta-\mathrm{SCF}$ approach (see section 2.5). Prior to the excitation, the system was heated to $300 \mathrm{~K}$ using standard ground-state molecular dynamics.

We follow the electron transfer by means of the Hirshfeld charge partitioning described in section 2.4 (see also refs 21 and 53). We partitioned the system into the slab representing the surface and the perylene molecule and observed an ultrafast transfer of $-0.2\left|q_{e}\right|$, which quickly saturates, as shown in Figure 5. After about $7 \mathrm{fs}$, the largest part of the transferred Hirshfeld charge settles on the $\mathrm{Ti}^{*}$ atom, that is, the $\mathrm{Ti}$ atom bonded to the oxygen of the carboxylic group. This is due to the effect of electron localization on Ti $d$-orbitals. After this time interval over which electron transfer occurs, the charge self-traps on this site by forming a surface polaron. This can be seen as a systematic shift of the average distance between $\mathrm{Ti}^{*}$ and the oxygen atoms to which it is bonded; at $\sim 60 \mathrm{fs}$, after the excitation, this distance increases by $0.07 \AA$ due to repulsion of the extra charge on $\mathrm{Ti}^{*}$. The polaron formation time of $\sim 60 \mathrm{fs}$ is in good agreement with the period of the $A_{1 g}$ phonon mode in rutile titania. ${ }^{86}$ Because of the formation of the polaron, the charge is trapped on $\mathrm{Ti}^{*}$ and further electron transfer is blocked. This may allow for high recombination rates with the hole located on the perylene. This charge transfer blockade due to the formation of the polaron makes rutile titania an inefficient substrate for dye and quantum dot sensitized solar cells. A more detailed analysis of the physics involved in these processes will be presented elsewhere.

3.5. Performance of the TDAP-2.0 Code. In Table 1, we present the computational resources required for the preceding demonstrations as well as values characterizing the size of each problem. All simulations were carried out on small number of cores (4 to 16) ${ }^{87}$ We found that a relatively large time step of 24.2 as $(1 \mathrm{au})$ is sufficiently accurate in most simulations; this is due to the self-consistent propagation scheme and refined matrix exponentiation routine. The last two columns in Table 1

Table 1. Performance of TDAP-2.0 Code. $N_{a t}$ is the number of atoms, $N_{e l}$ the number of valence electrons, $N_{b f}$ is the number of basis functions; $\Delta t$ is the time step, and $T_{t r a j}$ is the total simulated time

\begin{tabular}{|c|c|c|c|c|c|c|c|c|}
\hline system name & $N_{a t}$ & $N_{e l}$ & $N_{b f}$ & $\Delta t$ (as) & $T_{\text {traj }}(\mathrm{fs})$ & cores used & wall-clock time & core-hours \\
\hline AIBN & 24 & 64 & 216 & 24.2 & 194 & 4 & $8 \mathrm{~h} 31 \mathrm{~m} \mathrm{0s}$ & 34 \\
\hline $\mathrm{H} / \mathrm{Al}(111)$ & 73 & 217 & 652 & 20 & 80 & 16 & $8 \mathrm{~h} 26 \mathrm{~m} 53 \mathrm{~s}$ & 136 \\
\hline Fe porphyrin & 37 & 120 & 387 & 24.2 & 169 & 16 & $17 \mathrm{~h} 32 \mathrm{~m} 20 \mathrm{~s}$ & 280 \\
\hline perylene on $\mathrm{TiO}_{2}$ & 197 & 924 & 2465 & 24.2 & 115 & 16 & $9 \mathrm{~d} 8 \mathrm{~h} 05 \mathrm{~m} 25 \mathrm{~s}$ & 3585 \\
\hline
\end{tabular}


demonstrate the modest computational demands of our code for the reported simulations.

Although direct comparison of computational efficiency with other codes is difficult due to differences in the underlying implementation of DFT, we provide a crude level of comparison by simulating a few steps of the perylene on $\mathrm{TiO}_{2}$ with GPAW's classical ion dynamics. ${ }^{14}$ For the GPAW calculation, we use the default real-space grid spacing parameter $h=0.2 \AA$, use the same simulation cell and the same time step (24.2 as) as in our simulation but without imposing the selfconsistency of the propagation. From this run, we estimate the time required for a 115 fs trajectory to be 440,737 core-hours for GPAW, which translates to $>100$ times computationally more expensive than TDAP-2.0. This performance difference is expected $^{88}$ and is attributed to the use of the numerical atomicorbital basis set in contrast to the real-space grid approach employed by GPAW, the latter being better suited for highly accurate calculations of smaller systems.

In our simulation of perylene on rutile titania, the total energy was conserved within $14 \mathrm{meV}$ ( $0.07 \mathrm{meV}$ per atom). This is quite small considering all degrees of freedom in this simulation; it corresponds to a change of the system temperature by $0.4 \mathrm{~K}$. The deviation of total energy can be reduced by adjusting the parameters of the simulation, like the density matrix convergence cutoff in the self-consistency routine and the time step.

\section{CONCLUSIONS}

We presented a vastly improved and efficient version of our earlier approach for performing real-time first-principles electronic structure calculations, with classical ion dynamics and RT-TDDFT for the nonadiabatic propagation of the electronic system. The classical approximation for the electron-ion coupling still offers the best compromise between accuracy and tractability for the numerical simulation of large systems. This approximation, together with RT-TDDFT and utilization of localized, numerical atomic-orbital basis sets, allows for extremely efficient simulation of important problems such as photocatalysis and electron transfer on surfaces and interfaces. One of the most important additions to our method is the ability to use DFT $+U$ because it allows us to capture the effects of Coulomb repulsion and electron localization in transition metal oxide surfaces. These effects, combined with ionic motion, can have a profound influence on the behavior of the system, reaction outcomes, and electron transfer dynamics, as we have shown in examples here and in previous work. ${ }^{20} \mathrm{It}$ remains to be seen how much impact dynamic screening ("dynamic, site-specific $U$ ") and memory effects will have on the time evolution of such systems. We hope to address this issue in the future. The other major capability of our method is the simulation of linear absorption spectroscopy, where RTTDDFT has been shown to have unexpectedly good accuracy in reproducing experimental results. Last but not least is the addition of analysis tools, with the most important being the Hirshfeld charge partitioning method and the local dipole contributions. Our experience shows that this simple, intuitive, and stable method is remarkably well suited for analyzing the charge dynamics in spectroscopy, dye-sensitized solar cells and photocatalytic reactions. This can provide a qualitative picture of the process, which together with the low computational cost of our simulations could be of great utility in guiding experimental design of better materials for a variety of important applications. The computational tools developed here will hopefully be incorporated in standard packages like the SIESTA code that we have employed for their development. Before they become accessible as part of standard packages, they are available to academic researchers through collaboration by contacting the corresponding author.

\section{AUTHOR INFORMATION}

\section{Corresponding Author}

*E-mail: kaxiras@physics.harvard.edu.

Notes

The authors declare no competing financial interest.

\section{ACKNOWLEDGMENTS}

The work of G.K. was supported by Department of the Army, U.S. Army Research Office, through the Collaborative Research Alliance (CRA) for Multiscale Multidisciplinary Modeling of Electronic Materials (MSME). O.G. acknowledges the Swedish Research Council (VR) Grant No. 637-2013-7303 and the Ingegerd Bergh foundation for financial support. R.H. was supported as part of the Integrated Mesoscale Architectures for Sustainable Catalysis (IMASC), an Energy Frontier Research Center funded by the U.S. Department of Energy, Office of Science, Basic Energy Sciences under Award No. DESC0012573. The work of D.V. was supported by National Science Foundation Catalysis program under Grant No. CHE1362616. Computational resources were provided by XSEDE (Grant No. TG-DMR120073), which is supported by National Science Foundation Grant No. ACI-1053575, NERSC (ERCAP request number 88881 ) and the Odyssey cluster, supported by the FAS Research Computing Group at Harvard University.

\section{REFERENCES}

(1) Tully, J. C. Molecular dynamics with electronic transitions. J. Chem. Phys. 1990, 93, 1061-1071.

(2) Kohen, D.; Stillinger, F. H.; Tully, J. C. Model studies of nonadiabatic dynamics. J. Chem. Phys. 1998, 109, 4713-4725.

(3) Porezag, D.; Frauenheim, T.; Köhler, T.; Seifert, G.; Kaschner, R. Construction of tight-binding-like potentials on the basis of densityfunctional theory: Application to carbon. Phys. Rev. B: Condens. Matter Mater. Phys. 1995, 51, 12947.

(4) Elstner, M.; Porezag, D.; Jungnickel, G.; Elsner, J.; Haugk, M.; Frauenheim, T.; Suhai, S.; Seifert, G. Self-consistent-charge densityfunctional tight-binding method for simulations of complex materials properties. Phys. Rev. B: Condens. Matter Mater. Phys. 1998, 58, 7260.

(5) Pople, J. A.; Beveridge, D. L. Approximate Molecular Orbital Theory; McGraw-Hill: New York, 1970; Vol. 30.

(6) Dewar, M. J.; Thiel, W. Ground states of molecules. 38. The MNDO method. Approximations and parameters. J. Am. Chem. Soc. 1977, 99, 4899-4907.

(7) Ridley, J.; Zerner, M. An intermediate neglect of differential overlap technique for spectroscopy: pyrrole and the azines. Theor. Chim. Acta 1973, 32, 111-134.

(8) Barbatti, M.; Granucci, G.; Persico, M.; Ruckenbauer, M.; Vazdar, M.; Eckert-Maksić, M.; Lischka, H. The on-the-fly surface-hopping program system Newton-X: Application to ab initio simulation of the nonadiabatic photodynamics of benchmark systems. J. Photochem. Photobiol., A 2007, 190, 228-240.

(9) Zheng, J.; Li, Z.-H.; Jasper, A. W.; Bonhommeau, D. A.; Valero, R.; Meana-Pañeda, R.; Truhlar, D. G. ANT, version 2015. http:// comp.chem.umn.edu/ant.

(10) Richter, M.; Marquetand, P.; González-Vázquez, J.; Sola, I.; González, L. SHARC: Ab initio molecular dynamics with surface hopping in the adiabatic representation including arbitrary couplings. J. Chem. Theory Comput. 2011, 7, 1253-1258. 
(11) Du, L.; Lan, Z. An On-the-Fly Surface-Hopping Program JADE for Nonadiabatic Molecular Dynamics of Polyatomic Systems: Implementation and Applications. J. Chem. Theory Comput. 2015, $11,1360-1374$.

(12) Akimov, A. V.; Prezhdo, O. V. The PYXAID program for nonadiabatic molecular dynamics in condensed matter systems. J. Chem. Theory Comput. 2013, 9, 4959-4972.

(13) Akimov, A. V.; Prezhdo, O. V. Advanced capabilities of the PYXAID program: integration schemes, decoherence effects, multiexcitonic states, and field-matter interaction. J. Chem. Theory Comput. 2014, 10, 789-804.

(14) Ojanperä, A.; Havu, V.; Lehtovaara, L.; Puska, M. Nonadiabatic Ehrenfest molecular dynamics within the projector augmented-wave method. J. Chem. Phys. 2012, 136, 144103.

(15) Andrade, X.; Alberdi-Rodriguez, J.; Strubbe, D. A.; Oliveira, M. J.; Nogueira, F.; Castro, A.; Muguerza, J.; Arruabarrena, A.; Louie, S. G.; Aspuru-Guzik, A.; Rubio, A.; Marques, M. A. Time-dependent density-functional theory in massively parallel computer architectures: the OCTOPUS project. J. Phys.: Condens. Matter 2012, 24, 233202.

(16) Meng, S.; Kaxiras, E. Real-time, local basis-set implementation of time-dependent density functional theory for excited state dynamics simulations. J. Chem. Phys. 2008, 129, 054110.

(17) Meng, S.; Kaxiras, E.; Nazeeruddin, M. K.; Grätzel, M. Design of dye acceptors for photovoltaics from first-principles calculations. $J$. Phys. Chem. C 2011, 115, 9276-9282.

(18) Meng, S.; Kaxiras, E. Mechanisms for ultrafast nonradiative relaxation in electronically excited eumelanin constituents. Biophys. J. 2008, 95, 4396-4402.

(19) Soler, J. M.; Artacho, E.; Gale, J. D.; García, A.; Junquera, J.; Ordejón, P.; Sánchez-Portal, D. The SIESTA method for ab initio order-N materials simulation. J. Phys.: Condens. Matter 2002, 14, 2745.

(20) Kolesov, G.; Vinichenko, D.; Tritsaris, G. A.; Friend, C. M.; Kaxiras, E. Anatomy of the Photo-chemical Reaction: Excited State Dynamics Reveals CH Acidity Mechanism of Methoxy Photooxidation on Titania. J. Phys. Chem. Lett. 2015, 6, 1624.

(21) Hirshfeld, F. L. Bonded-atom fragments for describing molecular charge densities. Theor. Chim. Acta 1977, 44, 129-138.

(22) Saalmann, U.; Schmidt, R. Non-adiabatic quantum molecular dynamics: basic formalism and case study. Z. Phys. D: At., Mol. Clusters 1996, 38, 153-163.

(23) Doltsinis, N. L.; Marx, D. First principles molecular dynamics involving excited states and nonadiabatic transitions. J. Theor. Comput. Chem. 2002, 1, 319-349.

(24) Kunert, T.; Schmidt, R. Non-adiabatic quantum molecular dynamics: general formalism and case study $\mathrm{H} 2+$ in strong laser fields. Eur. Phys. J. D 2003, 25, 15-24.

(25) Andrade, X.; Castro, A.; Zueco, D.; Alonso, J. L.; Echenique, P.; Falceto, F.; Rubio, A. Modified Ehrenfest formalism for efficient largescale ab initio molecular dynamics. J. Chem. Theory Comput. 2009, 5, $728-742$.

(26) Runge, E.; Gross, E. K. U. Density-Functional Theory for TimeDependent Systems. Phys. Rev. Lett. 1984, 52, 997-1000.

(27) Castro, A.; Marques, M. A.; Rubio, A. Propagators for the timedependent Kohn-Sham equations. J. Chem. Phys. 2004, 121, 34253433.

(28) Riikonen, S. J.; Sánchez-Portal, D. First principles study of nanostructured surface reconstructions induced by the deposition of metals on vicinal Si(111) surfaces. Ph.D. Thesis, Universidad del País Vasco, Spain, 2007.

(29) Park, T. J.; Light, J. Unitary quantum time evolution by iterative Lanczos reduction. J. Chem. Phys. 1986, 85, 5870-5876.

(30) Sidje, R. B. Expokit: a software package for computing matrix exponentials. ACM Transactions on Mathematical Software (TOMS) 1998, 24, 130-156.

(31) Pulay, P. Ab initio calculation of force constants and equilibrium geometries in polyatomic molecules: I. Theory. Mol. Phys. 1969, 17, 197-204.

(32) Tully, J. Mixed quantum-classical dynamics. Faraday Discuss. 1998, 110, 407-419.
(33) Bornemann, F. A.; Nettesheim, P.; Schütte, C. Quantumclassical molecular dynamics as an approximation to full quantum dynamics. J. Chem. Phys. 1996, 105, 1074-1083.

(34) Li, X.; Tully, J. C.; Schlegel, H. B.; Frisch, M. J. Ab initio Ehrenfest dynamics. J. Chem. Phys. 2005, 123, 084106.

(35) Runge, E.; Gross, E. K. U. Density-Functional Theory for TimeDependent Systems. Phys. Rev. Lett. 1984, 52, 997-1000.

(36) Anisimov, V. I.; Zaanen, J.; Andersen, O. K. Band theory and Mott insulators: Hubbard U instead of Stoner I. Phys. Rev. B: Condens. Matter Mater. Phys. 1991, 44, 943.

(37) Anisimov, V. I.; Aryasetiawan, F.; Lichtenstein, A. I. Firstprinciples calculations of the electronic structure and spectra of strongly correlated systems: The LDA+U method. J. Phys.: Condens. Matter 1997, 9, 767-808.

(38) Czyżyk, M. T.; Sawatzky, G. A. Local-density functional and onsite correlations: The electronic structure of $\mathrm{La}_{2} \mathrm{CuO}_{4}$ and $\mathrm{LaCuO}_{3}$. Phys. Rev. B: Condens. Matter Mater. Phys. 1994, 49, 14211-14228.

(39) Petukhov, A. G.; Mazin, I.; Chioncel, L.; Lichtenstein, A. I. Correlated metals and the LDA+U method. Phys. Rev. B: Condens. Matter Mater. Phys. 2003, 67, 153106.

(40) Bultmark, F.; Cricchio, F.; Grånäs, O.; Nordström, L. Multipole decomposition of $\mathrm{LDA}+\mathrm{U}$ energy and its application to actinide compounds. Phys. Rev. B: Condens. Matter Mater. Phys. 2009, 80, 035121.

(41) Cococcioni, M.; de Gironcoli, S. Linear response approach to the calculation of the effective interaction parameters in the $\mathrm{LDA}+\mathrm{U}$ method. Phys. Rev. B: Condens. Matter Mater. Phys. 2005, 71, 035105.

(42) Kulik, H. J.; Marzari, N. Systematic study of first-row transitionmetal diatomic molecules: A self-consistent DFT $+U$ approach. J. Chem. Phys. 2010, 133, 114103.

(43) Aykol, M.; Wolverton, C. Local environment dependent GGA $+\mathrm{U}$ method for accurate thermochemistry of transition metal compounds. Phys. Rev. B: Condens. Matter Mater. Phys. 2014, 90, 115105 .

(44) Agapito, L. A.; Curtarolo, S.; Buongiorno Nardelli, M. Reformulation of DFT $+\mathrm{U}$ as a Pseudohybrid Hubbard Density Functional for Accelerated Materials Discovery. Phys. Rev. X 2015, 5, 011006.

(45) Lee, C.-C.; Hsueh, H. C.; Ku, W. Dynamical linear response of TDDFT with LDA+U functional: Strongly hybridized Frenkel excitons in NiO. Phys. Rev. B: Condens. Matter Mater. Phys. 2010, $82,081106$.

(46) Solovyev, I. V.; Liechtenstein, A.; Terakura, K. Is Hund's Second Rule Responsible for the Orbital Magnetism in Solids? Phys. Rev. Lett. 1998, 80, 5758-5761.

(47) Shick, A. B.; Drchal, V.; Havela, L. Coulomb-U and magneticmoment collapse in delta-Pu. Europhys. Lett. 2005, 69, 588-594.

(48) Liechtenstein, A. I.; Anisimov, V. I.; Zaanen, J. Densityfunctional theory and strong interactions: Orbital ordering in MottHubbard insulators. Phys. Rev. B: Condens. Matter Mater. Phys. 1995, 52, R5467-R5470.

(49) Dudarev, S. L.; Botton, G.; Savrasov, S. Y.; Humphreys, C.; Sutton, A. Electron-energy-loss spectra and the structural stability of nickel oxide: An LSDA+U study. Phys. Rev. B: Condens. Matter Mater. Phys. 1998, 57, 1505-1509.

(50) Tsolakidis, A.; Sánchez-Portal, D.; Martin, R. Calculation of the optical response of atomic clusters using time-dependent density functional theory and local orbitals. Phys. Rev. B: Condens. Matter Mater. Phys. 2002, 66, 235416.

(51) Lopata, K.; Govind, N. Modeling Fast Electron Dynamics with Real-Time Time-Dependent Density Functional Theory: Application to Small Molecules and Chromophores. J. Chem. Theory Comput. 2011, 7, 1344-1355.

(52) Pittalis, S.; Delgado, A.; Robin, J.; Freimuth, L.; Christoffers, J.; Lienau, C.; Rozzi, C. A. Charge Separation Dynamics and OptoElectronic Properties of a Diaminoterephthalate-C60 Dyad. Adv. Funct. Mater. 2015, 25, 2047-2053.

(53) Fonseca Guerra, C.; Handgraaf, J.-W.; Baerends, E. J.; Bickelhaupt, F. M. Voronoi deformation density (VDD) charges: 
Assessment of the Mulliken, Bader, Hirshfeld, Weinhold, and VDD methods for charge analysis. J. Comput. Chem. 2004, 25, 189-210.

(54) Ziegler, T.; Rauk, A.; Baerends, E. On the calculation of multiplet energies by the Hartree-Fock-Slater method. Theor. Chim. Acta 1977, 43, 261-271.

(55) von Barth, U. Local-density theory of multiplet structure. Phys. Rev. A: At., Mol., Opt. Phys. 1979, 20, 1693-1703.

(56) Gunnarsson, O.; Lundqvist, B. I. Exchange and correlation in atoms, molecules, and solids by the spin-density-functional formalism. Phys. Rev. B: Condens. Matter Mater. Phys. 1976, 13, 4274-4298.

(57) Gavnholt, J.; Olsen, T.; Engelund, M.; Schiøtz, J. $\Delta$ selfconsistent field method to obtain potential energy surfaces of excited molecules on surfaces. Phys. Rev. B: Condens. Matter Mater. Phys. 2008, $78,075441$.

(58) Casida, M. E. Time-dependent density-functional theory for molecules and molecular solids. J. Mol. Struct.: THEOCHEM 2009, 914, 3-18. Time-dependent density-functional theory for molecules and molecular solids.

(59) Dreuw, A.; Head-Gordon, M. Failure of time-dependent density functional theory for long-range charge-transfer excited states: The zincbacteriochlorin-bacteriochlorin and bacteriochlorophyll-spheroidene complexes. J. Am. Chem. Soc. 2004, 126, 4007-4016.

(60) Neugebauer, J.; Gritsenko, O.; Baerends, E. J. Assessment of a simple correction for the long-range charge-transfer problem in timedependent density-functional theory. J. Chem. Phys. 2006, 124, 214102.

(61) Onida, G.; Reining, L.; Rubio, A. Electronic excitations: densityfunctional versus many-body Green's-function approaches. Rev. Mod. Phys. 2002, 74, 601-659.

(62) Kowalczyk, T.; Yost, S. R.; Van Voorhis, T. Assessment of the $\triangle \mathrm{SCF}$ density functional theory approach for electronic excitations in organic dyes. J. Chem. Phys. 2011, 134, 054128.

(63) Görling, A. Density-functional theory beyond the HohenbergKohn theorem. Phys. Rev. A: At., Mol., Opt. Phys. 1999, 59, 3359-3374.

(64) Cullen, J.; Krykunov, M.; Ziegler, T. The formulation of a selfconsistent constricted variational density functional theory for the description of excited states. Chem. Phys. 2011, 391, 11-18. Open problems and new solutions in time dependent density functional theory.

(65) Gilbert, A. T.; Besley, N. A.; Gill, P. M. Self-Consistent Field Calculations of Excited States Using the Maximum Overlap Method (MOM). J. Phys. Chem. A 2008, 112, 13164-13171.

(66) Nguyen, N. L.; Borghi, G.; Ferretti, A.; Dabo, I.; Marzari, N. First-Principles Photoemission Spectroscopy and Orbital Tomography in Molecules from Koopmans-Compliant Functionals. Phys. Rev. Lett. 2015, 114, 166405 .

(67) Maurer, R. J.; Reuter, K. Excited-state potential-energy surfaces of metal-adsorbed organic molecules from linear expansion $\Delta$-selfconsistent field density-functional theory ( $\Delta$ SCF-DFT). J. Chem. Phys. 2013, 139, 014708.

(68) Newns, D. M. Self-Consistent Model of Hydrogen Chemisorption. Phys. Rev. 1969, 178, 1123-1135.

(69) Bird, D.; Persson, M.; Trail, J.; Holloway, S. Dynamics of the spin transition in the adsorption of hydrogen atoms on metals. Surf. Sci. 2004, 566-568 (Part 2), 761-766. Proceedings of the 22nd European Conference on Surface Science.

(70) Trail, J.; Bird, D.; Persson, M.; Holloway, S. Electron-hole pair creation by atoms incident on a metal surface. J. Chem. Phys. 2003, $119,4539-4549$.

(71) Lindenblatt, M.; Pehlke, E. Ab Initio Simulation of the Spin Transition during Chemisorption: $\mathrm{H} / \mathrm{Al}(111)$. Phys. Rev. Lett. 2006, 97, 216101.

(72) Lindenblatt, M.; van Heys, J.; Pehlke, E. Molecular dynamics of nonadiabatic processes at surfaces: Chemisorption of $\mathrm{H} / \mathrm{Al}(111)$. Surf. Sci. 2006, 600, 3624-3628. Berlin, Germany; September 4-9, 2005, Proceedings of the 23rd European Conference on Surface Science.

(73) Grätzel, M. Photoelectrochemical cells. Nature 2001, 414, 338344.

(74) Yella, A.; Lee, H. W.; Tsao, H. N.; Yi, C.; Chandiran, A. K.; Nazeeruddin, M. K.; Diau, E. W. G.; Yeh, C. Y.; Zakeeruddin, S. M.;
Grätzel, M. Porphyrin-Sensitized Solar Cells with Cobalt (II/III)Based Redox Electrolyte Exceed 12\% Efficiency. Science 2011, 334, 629-634.

(75) Hanson, E. K.; Ballantyne, J. A Blue Spectral Shift of the Hemoglobin Soret Band Correlates with the Age (Time Since Deposition) of Dried Bloodstains. PLoS One 2010, 5, 12830-12840.

(76) Wende, H.; Bernien, M.; Luo, J.; Sorg, C.; Ponpandian, N.; Kurde, J.; Miguel, J.; Piantek, M.; Xu, X.; Eckhold, P.; Kuch, W.; Baberschke, K.; Panchmatia, P. M.; Sanyal, B.; Oppeneer, P. M.; Eriksson, O. Substrate-induced magnetic ordering and switching of iron porphyrin molecules. Nat. Mater. 2007, 6, 516-520.

(77) Panchmatia, P. M.; Sanyal, B.; Oppeneer, P. M. GGA+U modeling of structural, electronic, and magnetic properties of iron porphyrin-type molecules. Chem. Phys. 2008, 343, 47-60.

(78) Luttrell, T.; Halpegamage, S.; Tao, J.; Kramer, A.; Sutter, E.; Batzill, M. Why is anatase a better photocatalyst than rutile?-Model studies on epitaxial $\mathrm{TiO}_{2}$ films. Sci. Rep. 2014, 4.10.1038/srep04043

(79) Yagi, E.; Hasiguti, R. R.; Aono, M. Electronic conduction above $4 \mathrm{~K}$ of slightly reduced oxygen-deficient rutile $\mathrm{TiO}_{2-x}$. Phys. Rev. B: Condens. Matter Mater. Phys. 1996, 54, 7945.

(80) Hendry, E.; Wang, F.; Shan, J.; Heinz, T. F.; Bonn, M. Electron transport in $\mathrm{TiO}_{2}$ probed by $\mathrm{THz}$ time-domain spectroscopy. Phys. Rev. B: Condens. Matter Mater. Phys. 2004, 69, 081101.

(81) Gundlach, L.; Ernstorfer, R.; Willig, F. Ultrafast interfacial electron transfer from the excited state of anchored molecules into a semiconductor. Prog. Surf. Sci. 2007, 82, 355-377.

(82) Ernstorfer, R.; Gundlach, L.; Felber, S.; Storck, W.; Eichberger, R.; Willig, F. Role of molecular anchor groups in molecule-tosemiconductor electron transfer. J. Phys. Chem. B 2006, 110, 2538325391.

(83) Tritsaris, G. A.; Vinichenko, D.; Kolesov, G.; Friend, C. M.; Kaxiras, E. Dynamics of the Photogenerated Hole at the Rutile $\mathrm{TiO}_{2}$ (110)/Water Interface: A Nonadiabatic Simulation Study. J. Phys. Chem. C 2014, 118, 27393-27401.

(84) Deskins, N. A.; Rousseau, R; Dupuis, M. Localized Electronic States from Surface Hydroxyls and Polarons in $\mathrm{TiO}_{2}(110)$. J. Phys. Chem. C 2009, 113, 14583-14586.

(85) Kowalski, P. M.; Camellone, M. F.; Nair, N. N.; Meyer, B.; Marx, D. Charge Localization Dynamics Induced by Oxygen Vacancies on the $\mathrm{TiO}_{2}(110)$ Surface. Phys. Rev. Lett. 2010, 105, 146405.

(86) Lan, T.; Tang, X.; Fultz, B. Phonon anharmonicity of rutile $\mathrm{TiO}_{2}$ studied by Raman spectrometry and molecular dynamics simulations. Phys. Rev. B: Condens. Matter Mater. Phys. 2012, 85, 094305.

(87) For all benchmarks described in this section, we used the Intel compiler suite 2015, Intel MKL math libraries, and -O2 optimization level on the XSEDE Stampede supercomputer.

(88) Kuisma, M.; Sakko, A.; Rossi, T. P.; Larsen, A. H.; Enkovaara, J.; Lehtovaara, L.; Rantala, T. T. Localized surface plasmon resonance in silver nanoparticles: Atomistic first-principles time-dependent densityfunctional theory calculations. Phys. Rev. B: Condens. Matter Mater. Phys. 2015, 91, 115431. 